\title{
Management of Acute Pulmonary Embolism
}

\author{
Connor Tice $^{1} \cdot$ Matthew Seigerman ${ }^{2,3,4}$ - Paul Fiorilli ${ }^{2,3,4} \cdot$ Steven C. Pugliese ${ }^{5}$. Sameer Khandhar ${ }^{6}$. Jay Giri $^{2,3,4,5}$. \\ Taisei Kobayashi ${ }^{2,3,4,5}$
}

Accepted: 28 September 2020 / Published online: 6 October 2020

(C) Springer Science+Business Media, LLC, part of Springer Nature 2020

\begin{abstract}
Purpose of the Review Over 100,000 cardiovascular-related deaths annually are caused by acute pulmonary embolism (PE). While anticoagulation has historically been the foundation for treatment of PE, this review highlights the recent rapid expansion in the interventional strategies for this condition.

Recent Findings At the time of diagnosis, appropriate risk stratification helps to accurately identify patients who may be candidates for advanced therapeutic interventions. While systemic thrombolytics (ST) is the mostly commonly utilized intervention for high-risk PE, the risk profile of ST for intermediate-risk PE limits its use. Assessment of an individualized patient risk profile, often via a multidisciplinary pulmonary response team (PERT) model, there are various interventional strategies to consider for PE management. Novel therapeutic options include catheter-directed thrombolysis, catheter-based embolectomy, or mechanical circulatory support for certain high-risk PE patients. Current data has established safety and efficacy for catheterbased treatment of PE based on surrogate outcome measures. However, there is limited long-term data or prospective comparisons between treatment modalities and ST. While PE diagnosis has improved with modern cross-sectional imaging, there is interest in improved diagnostic models for PE that incorporate artificial intelligence and machine learning techniques.

Summary In patients with acute pulmonary embolism, after appropriate risk stratification, some intermediate and high-risk patients should be considered for interventional-based treatment for PE.
\end{abstract}

Keywords Pulmonary embolism · Catheter-directed thrombolysis · Catheter-directed embolectomy $\cdot$ Pulmonary embolism response team

Connor Tice and Matthew Seigerman contributed equally to this work.

This article is part of the Topical Collection on Secondary Prevention and Intervention

Taisei Kobayashi

Taisei.kobayashi@pennmedicine.upenn.edu

1 Department of Internal Medicine, Hospital of the University of Pennsylvania, Philadelphia, PA 19104, USA

2 Division of Cardiovascular Medicine, Hospital of the University of Pennsylvania, 3400 Civic Center Blvd, Philadelphia, PA 19104, USA

3 Michael J. Crescenz Veteran Affairs Medical Center, Philadelphia, PA 19104, USA

4 Center for Cardiovascular Outcomes, Quality, and Evaluative Research, Philadelphia, PA, USA

5 Division of Pulmonology and Critical Care Medicine, Hospital of the University of Pennsylvania, Philadelphia, PA 19104, USA

6 Division of Cardiovascular Medicine, Penn Presbyterian Medical Center, Philadelphia, PA 19104, USA

\section{Introduction}

While pulmonary embolism (PE) results in more than 100,000 cardiovascular-related deaths annually in the USA (making it the 3rd leading cause of cardiovascular death behind myocardial infarction and stroke), there remains variability and limitations in evidence-based approaches to risk stratification and management of this preventable cause of death [1]. In fact, while there are perpetual attempts towards improved evidence-based approaches for the prevention and intervention of stroke and myocardial infarction, there is scant existing evidence for the management of venous thromboembolism across the wide spectrum of disease presentation despite it representing the single most preventable cause of in-hospital mortality [2]. While anticoagulation remains the mainstay of treatment for most cases of acute pulmonary embolism, given the elevated risk of mortality in those manifesting right ventricular (RV) dysfunction, there may be an opportunity to employ more aggressive therapies to improve outcomes in a 
carefully selected subset of acute PE patients [3, 4]. As a result, there is growing interest and shifting paradigms in regard to PE management. With accurate early risk stratification and appropriate patient selection via multidisciplinary approaches to management with the advent of pulmonary embolism response (PERT) teams, endovascular interventions may have the potential to reduce high rates of morbidity and mortality from acute PE.

\section{Risk Stratification}

The clinical presentation of acute PE can vary widely from asymptomatic, incidentally discovered emboli, to severe hemodynamic collapse and death $[5,6]$. Similarly, patients present with varying combinations of symptoms including dyspnea, chest pain, and respiratory compromise with limited clinical and examination techniques that can aid in the prediction of subsequent hemodynamic instability [6]. Given the broad spectrum of presentation, risk stratification utilizing multimodal assessment is of paramount importance. Two commonly used systems to classify PE are outlined by the American Heart Association (AHA) and the European Society of Cardiology (ESC) [7, 8•]. The AHA classifies acute PE into three categories: massive, submissive, and low risk [8•]. Similarly, the ESC categorizes PE into three categories: high risk, intermediate risk, and low risk.

- Massive (AHA) or high-risk (ESC) PE is defined similarly as emboli that lead to hemodynamic instability: systolic blood pressure $<90 \mathrm{mmHg}$, hypotension requiring vasopressor support, or a decrement of the systolic blood pressure $>40 \mathrm{mmHg}$ for at least $15 \mathrm{~min}[7,8 \cdot, 9]$. This cohort represents approximately $5 \%$ of patients with $\mathrm{PE}$ with inhospital mortality of approximately 22 to $30 \%$, approaching $65 \%$ in those requiring cardiopulmonary resuscitation [10].

- Submassive (AHA) or intermediate-risk (ESC) PE includes patients with right ventricular (RV) dysfunction in the absence of hypotension, as characterized by either CT angiography, echocardiography, or elevation in serum biomarkers (troponin or NT-proBNP). The ESC classification also denotes intermediate risk in the absence of right ventricular (RV) dysfunction in patients with a simple Pulmonary Embolism Severity Index (sPESI) score $\geq 1$, which aids in risk stratification of those with preexisting comorbid disease. The SPESI includes the following: age $>80$ years; cancer, chronic respiratory disease, or cardiac disease; heart rate $>110 \mathrm{bpm}$; systolic blood pressure $<100 \mathrm{~mm} \mathrm{Hg}$; or oxygen saturation $<90 \%$. The ESC classification further divides this group into intermediatehigh versus intermediate-low risk. Intermediate-high risks are emboli with both RV dysfunction and troponin elevation whereas intermediate-low risks are those patients with either RV dysfunction or troponin elevation. This cohort overall represents approximately $30 \%$ of patients with PE [10]. Prospective and retrospective observational data suggest mortality rates as high as $10 \%$ in the 3 months after the development of submassive- or intermediate-risk PE from registry data $[9,11,12]$.

- Low risk (AHA and ESC): All remaining patients that do not meet the criteria for submassive or intermediate risk are therefore classified as low risk. This cohort represents approximately $65 \%$ of patients with PE with 30-day mortality of approximately $1 \%[10,13]$.

Current data suggests that the volume of thrombus on presentation does not independently correlate with short-term mortality $[14,15]$. Therefore, a volumetric thrombus assessment is not a component of the above risk stratification strategies. Risk stratification is instead based more heavily on clinical assessment, objective markers of cardiac injury, and assessment of RV dysfunction given the correlation of these findings with short-term outcomes. Clinical manifestations of pulmonary embolisms are not static, and patients can quickly shift from one risk category to another. Thus, clinicians must rely on individualized and continual risk assessment for acute PE within hospitalized patients. The ultimate goal of risk stratification is to identify patients at high risk of mortality in order to identify potential candidates for more invasive and aggressive options for PE treatment. For the purposes of this review, we will utilize ESC criteria to define PE risk.

\section{Intervention of Acute Pulmonary Embolism}

Anticoagulation has been shown to be the foundation of treatment for most cases of PE [7]. Unless absolute contraindication for anticoagulation exists, prompt initiation of anticoagulation is important. Immediate initiation of aggressive anticoagulation at time of presentation is associated with a mortality benefit in comparison with those patients where time to therapeutic anticoagulation was delayed [16]. While anticoagulation alone has been shown to decrease RV/LV ratios by 0.12 in composite analysis of several RCTs, this degree of reduction is not known to correlate with a significant difference in prevention of clinical decompensation in intermediate-risk patients [17]. Subsequent adverse outcomes in such patients have sparked interest in advanced therapeutic strategies.

The aim of this review is to discuss the current options for advanced therapies for pulmonary embolism including systemic thrombolytics and catheter-directed interventions. As described previously, most patients across the wide spectrum of PE presentations experience low-risk PE with an associated low mortality. In these patients, a strategy of anticoagulation 
alone should be pursued. More advanced interventions can be considered for patients with intermediate- or high-risk PE. However, these patients compromise a smaller proportion of patients with PE, and as such, recruitment for high-quality randomized trials has proved challenging. Furthermore, due to the complexity of rigorously studying high-risk PE patients, the majority of data studying the efficacy of catheter-directed interventions in $\mathrm{PE}$ is from single-arm prospective trials in patients with mostly intermediate-risk PE subsequently affecting the generalizability of these results to all patients presenting with PE. These considerations must be taken into account when subjecting patients to higher risk advanced therapies as comparative effectiveness data for these clinical interventions is relatively limited. This review aims to disclose the benefits and risks of each of these strategies to help with clinical decision-making.

\section{Systemic Thrombolysis}

Systemic thrombolysis (ST) refers to the administration of a pharmacologic thrombolytic agent via a peripheral intravenous line. The benefit of ST involves swift improvement of pulmonary artery pressures, RV function, ventilation/ perfusion matching, clinical hemodynamic status, and clinical symptoms $[7,8 \bullet]$. This benefit is balanced against and elevated risk of major bleeding (including intracranial hemorrhage) compared with anticoagulation alone [18]. Historically, ST was the treatment of choice for high-risk PE given the acute nature of these presentations and risk for further rapid deterioration [7, 8•]. In the PEITHO trial, 1006 intermediate-risk patients were randomized to ST versus anticoagulation alone in order to evaluate a composite outcome of short-term allcause mortality or hemodynamic collapse within 7 days [19]. ST prevented this composite primary outcome (2.6\% versus $5.6 \% ; p=0.015)$ compared with anticoagulation alone. Subgroup analysis revealed this was mainly influenced by an increased rate of hemodynamic decompensation with anticoagulation ( 1.6 versus $5.0 \% ; p=0.002)$ rather than mortality. However, the study was underpowered to detect a true difference in mortality. Major bleeding and stroke were both observed more frequently in the ST group compared with anticoagulation alone $(6.3 \%$ versus $1.2 \% ; p<0.001)$ and ( $2.4 \%$ versus $0.2 \% ; p=0.003)$, respectively. Subsequent meta-analysis data from Chatterjee and colleagues demonstrated modest mortality rate reduction with ST use in intermediate-risk PE (2.17\% versus $3.89 \%$, NNT 59) in comparison with anticoagulant therapy, at the expense of increased rates of major bleeding and intracerebral hemorrhage (major bleeding: $9.24 \%$ versus $3.42 \%$, NNH 18; ICH: $1.46 \%$ versus $0.19 \%$, NNH 78 [12]. Given lack of significant mortality benefit and higher rates of major bleeding, the 2016 CHEST guidelines recommended against universal ST therapy in all intermediate-risk PEs [19, 20]. Overall, data on ST suggests that thrombolytic therapy is efficacious, yet bleeding risk must be reduced in order to optimize the benefit to risk ratio for this therapy.

\section{Catheter-Directed Thrombolysis}

In line with the ethos to decrease bleeding risk to the patient, catheter-directed thrombolysis (CDL) involves the direct injection of pharmacologic thrombolytic agents into the pulmonary artery vasculature with the goal to minimize systemic distribution and theoretically reduce the risk of major bleeding events. Non-ultrasound-assisted (non-us) CDL commonly utilizes the Uni-Fuse (AngioDynamics Inc., Latham, NY) or Cragg-McNamara (ev4 Inc., Plymouth, MN) catheters. Both devices have a Food and Drug Administration (FDA) indication for treatment within the peripheral vasculature via the 510(k) clearance but lack a specific indication for PE. Typically, 4 or $5 \mathrm{Fr}$ catheters with infusion lengths of 5 to $10 \mathrm{~cm}$ are advanced to a position within a pulmonary thrombus. Thus far, there is sparse data evaluating traditional CDL system use within the pulmonary vasculature. The PERFECT study from Kuo and colleagues examined the utility of CDL versus mechanical thrombectomy for high-risk $(n=28)$ and intermediate-risk $(n=73)$ patients utilizing registry data [21]. The CDL group was a mixture of both non-us CDL (64\%) and ultrasound-assisted thrombolysis (USAT) (36\%). Among those who underwent CDL, there were no reported major bleeding events and a $5.9 \%$ in-hospital mortality rate.

The only FDA-approved CDL catheter remains the EKOSonic endovascular system (EKOS Corp, Bothell, WA). This system utilizes ultrasound-assisted thrombolysis (USAT) via a dual-lumen 5Fr catheter, in which one lumen facilitates delivery of local thrombolytic therapy and a second lumen that facilitates the delivery of a separate ultrasound emitting transducer (Fig. 1). The utilization of ultrasound technology is thought to facilitate fibrin strand dissociation and accelerate local delivery of pharmacologic thrombolytics [22]. USAT was originally studied in two prospective studies, ULTIMA and SEATTLE II. Both studies showed improvement in the surrogate outcome of $\mathrm{RV} / \mathrm{LV}$ ratio within 24 and $48 \mathrm{~h}$, respectively [23, 24]. Multicenter retrospective studies comparing USAT with non-us CDL in intermediate- and high-risk PE patients demonstrated equivalent clinical effectiveness with significant right ventricular offloading; however, they did not demonstrate short- or long-term benefit in regard to functional quality of life metrics or safety endpoints such as mortality or periprocedural complication rates $[21,25$, $26 \bullet, 27]$. While these initial studies appear to favor the use of USAT, direct prospective comparisons with non-us CDL are underway. 


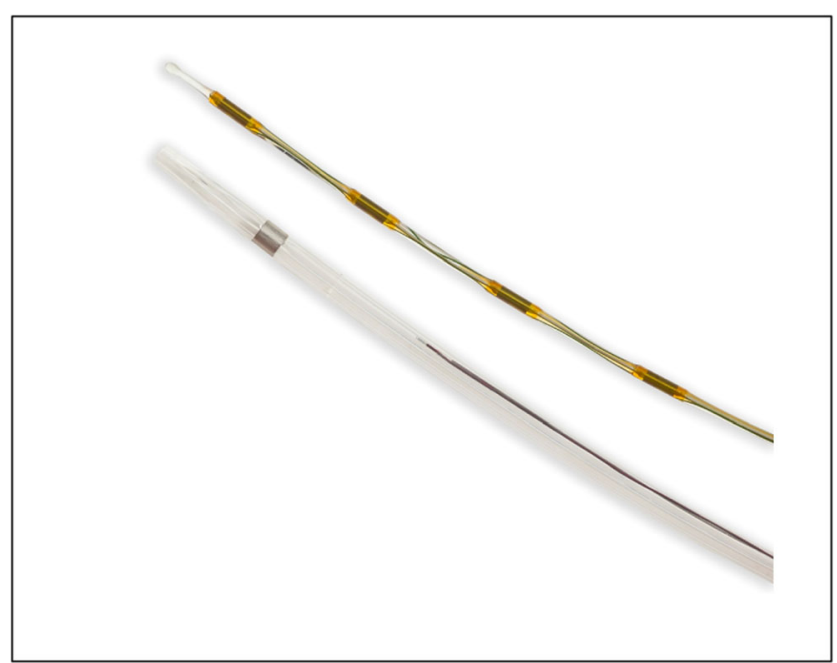

Fig. 1 EKOSonic ultrasound-assisted thrombolysis system. Image provided courtesy of Boston Scientific. (C2020 Boston Scientific Corporation or its affiliates. All rights reserved

In addition, the optimal duration and dose of CDL, independent of ultrasound assistance, has not been well studied. Trials have used a variety of infusion doses ranging from 1 to $2 \mathrm{mg}$ per h, for durations ranging from 2 to $24 \mathrm{~h}$ of infusion time (Table 1) [21, 23, 24, 28•]. In theory, the lower the dose of thrombolytic drug should translate to lower rates of bleeding complications such as intracerebral hemorrhage (ICH). While reformulated and novel risk scores have been created for intracerebral hemorrhage with thrombolytic use, a dosedependent relationship has yet to be definitively shown [29, 30]. At present, a pooled analysis of all prospective studies of CDL demonstrate a $0.7 \%$ rate of ICH $[31 \bullet \bullet$. While not an apples to apples comparison, a prior study of prospective studies of ST reported a rate of $1.5 \%$ [12]. There are currently no published, randomized, prospective studies directly comparing USAT and ST; however, three are underway (NCT03086317, NCT02758574, and NCT03389971). Therefore, the previously discussed data must be interpreted cautiously.

Additionally, data available to evaluate long-term benefits of CDL therapies has been challenging to interpret. While several single-center retrospective trials have evaluated USAT versus either anticoagulation alone or non-us CDL with longer term endpoints (e.g., 12 months), such study design subjects these trials to significant bias and limit the generalizability of the results to relevant clinical populations [25, $26 \cdot, 32]$. Thus, while limited high-quality data exist on the use of CDL in acute PE, it appears to have a lower rate of overall bleeding and ICH in comparison with ST in acute PE with encouraging outcomes at 30 days. Prompt decision-making regarding ST versus catheter-directed approaches remains difficult, and often, the decision is provoked by relative or absolute contraindications as opposed to clear indication or preference for one treatment modality. Overall dose and durations are still under active research as well as longer term benefits to this strategy.

\section{Catheter-Based Embolectomy}

Catheter-based embolectomy is distinct from CDL and refers to mechanical thrombus disruption and removal in an effort to
Table 1 Comparison between catheter-directed thrombolysis trials in regard to device investigated, dose of pharmacologic thrombolysis used, and surrogate outcome based on right ventricle (RV)/left ventricle (LV) reduction [note: $\mathrm{RV} / \mathrm{LV}$ reduction with heparin alone historically 0.12 .
USAT, ultrasound-assisted thrombolysis; non-us $C D L$, non-ultrasoundassisted catheter-directed thrombolysis; rtPA, recombinant tissue plasminogen activator; $t P A$, tissue plasminogen activator; ITT, intention to treat

\begin{tabular}{|c|c|c|c|c|c|c|c|}
\hline Trial & $N$ & Device & Study type & Dose & Duration (h) & Max dose & $\begin{array}{l}\mathrm{RV} / \mathrm{LV} \\
\text { reduction }\end{array}$ \\
\hline $\begin{array}{l}\text { ULTIMA } \\
{[23]}\end{array}$ & $\begin{array}{l}59 \text { (30 with } \\
\text { USAT) }\end{array}$ & USAT & RCT & $\begin{array}{l}\mathrm{rtPA} @ 1 \mathrm{mg} / \mathrm{h} \text { for } 5 \mathrm{~h}, \\
0.5 \mathrm{mg} / \mathrm{h} \text { for } 10 \mathrm{~h}\end{array}$ & $15 \pm 1$ & $\begin{array}{l}20 \pm 1 \text { mg bilateral; } 10 \pm 0.5 \\
\text { mg unilateral }\end{array}$ & $\begin{array}{l}0.35 \pm 0.22 @ \\
24 \mathrm{~h}\end{array}$ \\
\hline $\begin{array}{l}\text { SEATTLE II } \\
{[24]}\end{array}$ & 150 & USAT & $\begin{array}{l}\text { Prospective, } \\
\text { single-arm }\end{array}$ & $1 \mathrm{mg} / \mathrm{h}$ & $\begin{array}{l}24 \text { unilateral; } \\
\text { 12-bilateral }\end{array}$ & $24 \mathrm{mg}$ & $\begin{array}{l}0.42 \pm 0.36 @ \\
\quad 48 \mathrm{~h}\end{array}$ \\
\hline \multirow[t]{4}{*}{$\begin{array}{l}\text { OPTALYSE } \\
{[28 \bullet]}\end{array}$} & 101 & USAT & $\mathrm{RCT}$ & $4-12 \mathrm{mg}$ & $2-6$ & $8-24 \mathrm{mg}$ & $\begin{array}{l}\text { ITT @ } 48 \text { h } \\
\text {-Arm 1: } \\
\quad 24.0 \pm 15.9\end{array}$ \\
\hline & & & & & & & $\begin{array}{l}-\operatorname{Arm} 2: \\
\quad 22.6 \pm 14.1\end{array}$ \\
\hline & & & & & & & $\begin{array}{l}-\operatorname{Arm} 3: \\
\quad 26.3 \pm 16.8\end{array}$ \\
\hline & & & & & & & $\begin{array}{l}-\operatorname{Arm} 4: \\
\quad 25.5 \pm 22.7\end{array}$ \\
\hline $\begin{array}{l}\text { PERFECT } \\
{[21]}\end{array}$ & 101 & $\begin{array}{l}\text { Non-us CDL } \\
\text { and USAT }\end{array}$ & $\begin{array}{l}\text { Prospective } \\
\text { registry }\end{array}$ & $\begin{array}{l}\text { tPA } 0.5-1 \mathrm{mg} / \mathrm{h} \text { OR } \\
\quad \text { urokinase } 100,000 \mathrm{IU} / \mathrm{h}\end{array}$ & Unspecified & $\begin{array}{l}\text { Average dose tPA } 28.0 \pm 11.0 \mathrm{mg} \\
2,697,101 \pm 936,287 \text { IU urokinase }\end{array}$ & Not reported \\
\hline
\end{tabular}


decrease thrombotic burden rather than relying solely on pharmacologic effects of thrombolytics. Percutaneous mechanical interventions for thrombus disruption and aspiration were previously considered in tenuous, non-ST candidates either due to concern for hemorrhagic complications or degree of thrombotic burden. Currently, however, such therapies are now being considered a primary reperfusion strategy, or in conjunction with local pharmacologic thrombolysis.

\section{Rheolytic Thrombectomy}

The AngioJet system (Boston Scientific, Minneapolis, MN) utilizes $6 \mathrm{Fr}$ - to $8 \mathrm{Fr}$-sized catheters and relies on the Bernoulli principle to generate a pressure gradient utilizing highpressure saline jets at the tip of the catheter with subsequent area of low pressure designed for aspiration of thrombus. Local low-dose thrombolytic can be utilized instead of saline to provide thrombolysis in conjunction with local disruption [33]. Despite evidence of feasibility in multiple case series, the Angiojet system was associated with significant bradyarrhythmia, hypotension, and hypoxia due to presumed pulmonary hemorrhage with subsequent circulatory collapse $[34,35]$. Subsequent animal studies attributed these complications to significant vasoreactivity from AngioJet catheter placement resulting in significant release of bradykinin and adenosine [36]. While this device continues to have $510(\mathrm{k})$ clearance from the FDA for peripheral thrombus, the aforementioned concerns led to an FDA black box warning for intrapulmonary use and should be avoided.

\section{Large Bore Catheter-Based Embolectomy}

The FlowTriever system (Inari Medical, Irvine, CA) utilizes large bore femoral venous access (20-24Fr), with an aspiration guide catheter (AGC) advanced near the thrombus. The clot is removed primarily by creating a negative suction through the AGC. Often, several aspirations are needed for larger or more chronic clots. This device also has the additional benefit of combining aspiration clot removal with a mechanical mechanism. A catheter consisting of three nitinol disks can be passed through the AGC and deployed within or distal to the clot. Retraction of the disks combined with aspiration allows for removal of more organized or distal clot. This system obtained its FDA 510(k) clearance for PE after the publication of the FLARE trial (FlowTriever Pulmonary Embolectomy Clinical Study), a prospective, single-arm, multicenter investigational device exemption study [37•]. This study included 106 patients with acute, intermediate-risk PE, best defined by $\mathrm{RV} / \mathrm{LV}$ ratio $>0.9$ on $\mathrm{CT}$ angiography in the absence of hemodynamic instability. However, $55.8 \%$ had a sPESI score $=0$, and elevated troponin and NT-proBNP were evident in $59.6 \%$ and $72.4 \%$ of patients, respectively. In an effort to study the efficacy and safety of mechanical thrombectomy in isolation, this study excluded those who received pharmacologic thrombolysis. In terms of clinical effectiveness, there was a significant reduction in the RV/LV ratio at $48 \mathrm{~h}$ post-procedure by 0.38 , equating to an averaged reduction of $25.1 \%$, while modest invasive mean PA pressure reduction was only evident in those with pulmonary hypertension at time of presentation [37•]. In terms of safety, bleeding events were defined by Valve Academic Research Consortium-2 (VARC-2) guidelines. Protocol-defined bleeding occurred in $1.0 \%$ of cases, and $3.8 \%$ of patients suffered other major adverse events including treatment-related clinical deterioration, pulmonary vascular injury, or cardiac injury within $48 \mathrm{~h}$ of thrombectomy. Despite an expected technical learning curve with the majority of enrolling sites performed less than 5 cases, there was no site heterogeneity identified in regard to effectiveness or safety outcomes in this early experience with the Flowtriever [37॰]. Thus, for large bulky proximal thrombi and higher bleeding risk patients, this appears to be a preferable strategy with the caveat that these larger devices may be associated with a higher risk of periprocedural hemodynamic or pulmonary collapse due to the size of the device. At present, this is the only large bore embolectomy system cleared by the FDA for pulmonary embolism. Further studies of this device in intermedate- and massive-risk PEs are underway.

The AngioVac (Angiodynamics, Inc.) is an extracorporeal veno-veno bypass system designed with a filter for en block suction thrombectomy $[38,39]$. The design includes extracorporeal filtration of intravascular debris utilizing a funnelshaped inflow tip to engage intravascular thrombi and a reinfusion catheter to mitigate blood loss. The inflow catheter is $22 \mathrm{Fr}$ and can be utilized via femoral or internal jugular approach, while the outflow cannula is $16-20 \mathrm{Fr}$ via alternative access [39]. Several single-center studies have been published, particularly including patients with extensive caval thrombi, intracardiac thrombus, or thrombus-in-transit in the context of PE, with very limited data on its efficacy and safety in the pulmonary vasculature. The major anatomic disadvantage of early generations of this device related to the relative unwieldiness of navigating the inflow cannula into the pulmonary arterial circulation. Newer iterations of this system have focused on improved operator navigation into the right ventricular outflow tract for more targeted intervention for PE. However, prospective studies designed to obtain FDA clearance for use in the pulmonary vasculature have not yet been initiated.

\section{Small Bore Catheter-Based Embolectomy}

The Indigo Thrombectomy System (Penumbra, Inc., Alameda, CA) utilizes an $8 \mathrm{Fr}$ aspiration catheter with a 
continuous vacuum pump that allows for mechanized aspiration. The benefit to utilization of this device in comparison with the Flowtriever system is its relative size which would theoretically decrease the chance of hemodynamic collapse. However, this may also limit aspiration of larger bulky thrombi. A larger 12 French device will be available soon to allow for removal of larger clots. In addition, serial monitoring of the collecting chamber is imperative, as continuous suction results in acute blood loss, currently without the ability to recycle aspirated blood [40]. Two pre-existing single-center series including 24 patients reported no intraprocedural decompensations; however, three episodes of major bleeding including ICH were noted, although this was in the context of concomitant thrombolytic use [41, 42]. EXTRACT-PE (NCT03218566), a prospective, single-arm, investigational device exemption trial, has completed recruitment and reported initial results in oral presentation form, evaluating its use in intermediate-risk PE (RV/LV ratio $>0.9$ in the absence of hypotension or shock). Similar to the FLARE trial, the primary efficacy endpoint was RV/LV ratio reduction, with safety profile assessment based on a composite that included devicerelated death, major bleeding, and device-related serious adverse events. In this study, there was a $27.3 \%$ reduction in RV/ $\mathrm{LV}$ ratio at $48 \mathrm{~h}$ post-procedure, with a low major adverse outcome event rate of $1.7 \%$ within $48 \mathrm{~h}$; however, details in regard to this clinical endpoint are limited [43]. Further publication and assessment of its effectiveness is pending. As a result of this preliminary data, the Indigo Thrombectomy system received FDA 510(k) clearance for treatment of PE as of January 2020 [44]. The three FDA-approved devices are shown in Figs. 1, 2, and 3.

Several of the devices discussed have promising application within the pulmonary vasculature for treatment of acute PE. At present, the 2016 CHEST guidelines, catheter-based mechanical embolectomy without thrombolysis was only considered in high-risk patients in shock who have high bleeding risk or who failed thrombolysis; however, we expect future guidelines to broaden the indications for the use of these catheter-directed interventions based upon these recent studies [20].

The evolution of percutaneous therapies for the acute management of PE has adapted to the spectrum of disease presentations and associated comorbidities that may alter decisionmaking. With the advent of catheter-directed thrombolysis and catheter-based embolectomy, two distinct pharmacomechanical therapies have garnered interest with increasing use. While the benefit of catheter-directed thrombolytic therapy relies upon the theoretical improvement in major bleeding events through local administration, such complications remain with current devices. Concurrently, catheterbased embolectomy devices promote thrombus disaggregation and may provide value as PE risk increases; however, such interventions carry higher rates of intraprocedural risk due to catheter size and limited maneuverability that may exacerbate cardiac or pulmonary vascular trauma. Hybrid strategies utilize novel interventional catheters such as the Bashir Endovascular Catheter. The unique design of this apparatus includes six expandable infusion limbs that create a short basket that serves the dual function of thrombus disaggregation and improved radial dispersion of catheter-directed thrombolysis to a larger surface area of exposed thrombus. Feasibility and safety studies are underway for this integrated approach at pharmacomechanical means of PE management (NCT03927508).

\section{Mechanical Circulatory Support}

Mechanical cardiopulmonary support in the form of venoarterial (VA) extracorporeal membrane oxygenation (ECMO) may be an intervention temporarily deployed for patients with high-risk PE with cardiogenic shock or cardiac arrest. Given this circuit bypasses the pulmonary vasculature, there is a significant reduction of the RV preload and reduction of RV dilation. Currently, no guidelines or consensus statements exist to guide the use of ECMO in cases of acute PE with no randomized controlled trials available to truly assess efficacy or safety. Thus far, data supporting its use is limited to case series for patients suffering high-risk PE with circulatory collapse or cardiac arrest. While ECMO as a standalone therapy with anticoagulation has been proposed [45], other reports suggest caution with this approach and suggest its use alongside other therapeutic interventions such as CDL and mechanical thrombectomy $[8 \bullet, 46 \bullet]$. In one retrospective analysis by Ain and colleges, after the implementation of ECMO in a hospital system, 30-day survival for all cases of high-risk PE, regardless of ECMO use, significantly improved compared with those treated before ECMO was available (17.2 versus $41.4 ; p=0.043$ ) [47]. Overall, 30-day survival rates from several case series for high-risk PE with the use of ECMO is estimated at $31-61.5 \%[46 \cdot, 48]$. In a patient with active critical deterioration occurring before targeted therapy is implemented, ECMO can provide temporary hemodynamic and oxygenation support, thereby serving as a bridge facilitating the implementation of other advanced interventions [47, 49].

\section{Pulmonary Embolism Response Teams}

Despite increasing clinical appreciation for appropriate risk stratification of acute $\mathrm{PE}$, gaps in knowledge remain in treatment of high-risk and intermediate-risk PE, with low utilization of thrombolytic therapy either systemically or through catheter-directed approaches as more patients present with absolute or relative contraindications to 
Fig. 2 a FlowTriever embolectomy system (Inari Medical, Irvine, CA). b FlowTriever catheter. c Cather engaged during active thrombus removal. Images provided courtesy of Inari Medical. ()2020 Inari Medical. All rights reserved

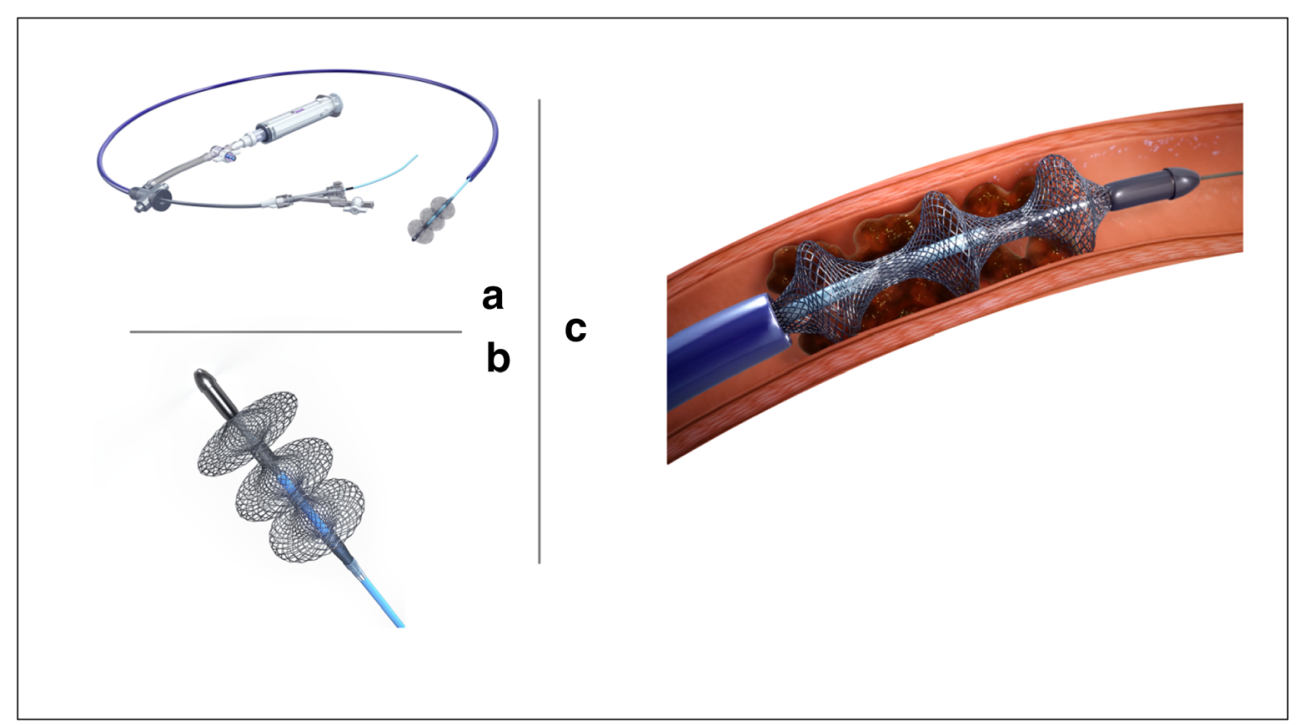

thrombolytic therapy $[6,50]$. Over the past decade, pulmonary embolism response teams (PERTs) have been established akin to trauma and stroke evaluation teams, given the time-sensitive nature of clinical deterioration and nuanced decision-making required for subsequent intervention [51]. PERTs rely upon a multidisciplinary approach, most often integrating clinical decision-making across pulmonary/critical care (84\%), interventional cardiology $(79 \%)$, and emergency medicine $(63 \%)$, and can often include members from cardiac surgery, hematology, and vascular medicine $[52,53]$. While there is variability in real-time multidisciplinary assessment, the inclusion of providers from across the health care system provides an infrastructure to efficiently mobilize resources and creates a mechanism for internal quality assessment and improvement. Given the heterogeneity in regard to clinical presentation and management of acute PE, a PERT approach that is inclusive of varied medical disciplines reflects the diversity of the clinical experience. This mod$\mathrm{el}$, in comparison with similar models for shock, cardiac arrest, or trauma, may have even more profound impact given the paucity of data. To address the lack of data, the development of the National PERT Consortium has been created as a platform for dynamic collaboration, consensus guidelines, and a centralized database that will allow for retrospective post-market surveillance of catheterdirected approaches, while also gleaning evidence for changes to clinical endpoints in future prospective studies. Centralization of data and knowledge locally via PERT and nationally at the PERT consortium may play a large
Fig. 3 Penumbra system - a Penumbra ENGINE ${ }^{\mathrm{TM}}$ aspiration source. b Indigo system CAT8 mechanical thrombectomy catheters. Images provided courtesy of Penumbra Inc. (C2020 Penumbra Inc. All rights reserved

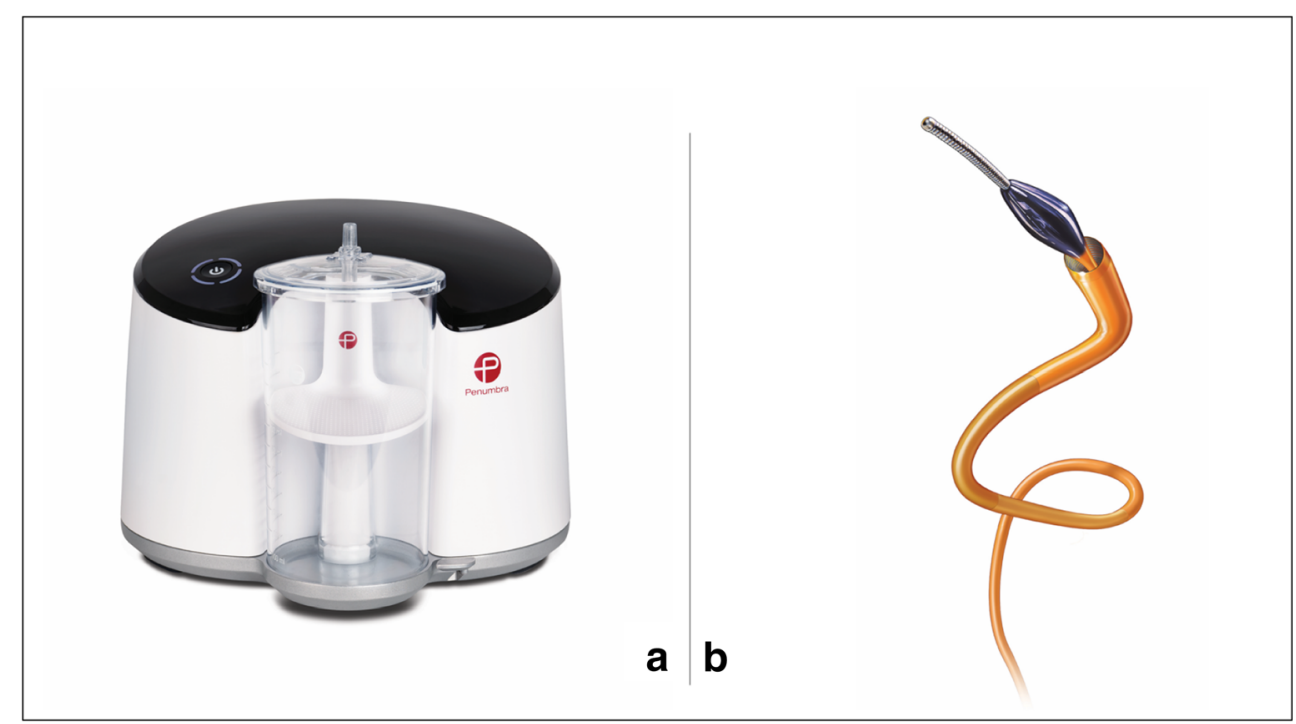


role for the future of clinical decision-making in PE which will be discussed later $[54 \bullet \bullet, 55]$.

\section{Limitations to Current Data in PE and Future Directions}

The major limitation of most clinical trials to date evaluating advanced therapeutic options for PE is that they are underpowered for important long-term outcome and safety measures. Most trials studying efficacy and safety data come from small single-arm prospective data sets which may limit the ability to guide generalized clinical practice. The clinical endpoint for most of these trials has remained the $\mathrm{RV} / \mathrm{LV}$ ratio as a surrogate marker for mortality based upon extrapolated observational data that has demonstrated correlations with 30-day mortality [56]. It remains unclear if this surrogate marker translates to longer term outcomes. Furthermore, there is an unmet need for expansion of clinical endpoints in $\mathrm{PE}$ trials to include patient-centered outcomes as primary endpoints, including objective functional assessment (e.g., dyspnea evaluation, 6 Minute Walk Test (6MWT), New York Heart Association (NYHA) classification, cardiopulmonary exercise testing (CPET)) in order to appropriately evaluate the mid and long-term impact of catheter-directed approaches to PE on the morbidity associated with this disease [57].

\section{The Impact of Rising Trends in PE Diagnosis and Management on Percutaneous Strategies}

As discussed throughout this review, prompt diagnosis and risk stratification in PE can lead to life-saving intervention; however, risk modeling remains a moving target. Due to the heterogeneity of the data, real-time incorporation of artificial intelligence and machine learning techniques may aid in objective quantification of risk for PE and subsequent risk stratification utilizing electronic health record and imaging data. Banerjee and colleagues created the Pulmonary Embolism Result Forecast Model (PERFORM) to aid in predicting the diagnosis of $\mathrm{PE}$, aimed at curbing inappropriate crosssectional imaging utilization [58•]. By parsing through raw data including inpatient and outpatient medications, demographic information, prior diagnosis codes, and raw data from laboratory testing, this group created a deep neural network model that achieved an area under the receiver operating characteristic curve (AUROC) performance of predicting a positive PE study as high as 0.90 (95\% CI, 0.87-0.91). Models like PERFORM can be incorporated in parallel with current triage strategies and automatically provide patient-specific risk for PE that may allow for earlier recognition and treatment for those with high positive PE prediction scores. While targeted for identifying optimal candidates for imaging, future models can incorporate real-time data from either cross-sectional imaging or echocardiography to help identify patients who would benefit from intervention in a timely fashion for suspected PE.

While advances in this area are in its infancy, there have been initial advances in deep learning techniques to help utilize the pre-existing data that all patients with PE will have at the time of diagnosis. Current practice in the radiographic interpretation of $\mathrm{CT}$ angiography for suspected $\mathrm{PE}$ is to generate a binary response, promptly identifying the presence or absence of PE. Pre-existing data exists to support risk stratification by thrombus location (e.g., central versus peripheral) [59]. Additionally, models do exist to quantify clot burden utilizing segmentation assessment and generation of semiquantitative scores (e.g., Qanadli and Mastora scores); however, these modalities are time-consuming and have moderate interreader variability. Liu and colleagues incorporated deep learning algorithms in the reading of $\mathrm{CT}$ angiography for PE and were able to correlate the degree of clot burden and RV parameters such as $\mathrm{RV} / \mathrm{LV}$ ratio and $\mathrm{PA}$ dimensions, with the contemporary, time exhaustive techniques of generating Qanadli and Mastora scores [60]. Qanadli and Mastora score generation has been shown to correlate with degree of right ventricular dysfunction; however, these models have not been shown to correlate with adverse clinical outcomes including mortality [61-63]. Thus, while this model may provide prompt identification of RV dysfunction that may classify a patient with at least intermediate-risk PE, there is lacking data to support its impact to unilaterally providing prognostic value. This study and the current literature only skim the surface of the potential of artificial intelligence and machine learning techniques on timely, objective, and quantifiable means to identify PE and its associated risk.

Neural network and deep machine learning incorporation into ongoing efforts at the National PERT Consortium would not only provide clinical data to support or refute current clinical practice but will aid in judicious and appropriate use of interventional strategies in patients newly identified as high risk beyond current means of risk stratification. One potential practical application for these tools is in the diagnosis of venous thromboembolism (VTE) in patients with COVID-19. By some estimates, the incidence of VTE in these populations approaches $30 \%$ in patients treated in a critical care setting [64]. This high rate of VTE and subsequent PE may be one key driver of the increased mortality in these patients. However, diagnostic challenges exist with these patients 
given resource allocation limitations, clinical staff exposure risk, and challenging illness factors to navigate (e.g., severe ARDS necessitating prone positioning) that may limit conventional diagnostic approaches to PE. The application of neural networks and deep machine learning potentially could assist clinicians attempting to diagnose thrombotic complications in these patients and implement therapies before the critical development of right ventricular dysfunction. When VTE is diagnosed, society guidelines stress the importance of individualized risk stratification [65]. While early guidance suggests anticoagulation should remain the mainstay of therapy for patients with COVID-19, given potential drug interactions with disease targeted therapy and anticoagulation, there may be a role for advanced therapeutics in critical scenarios [65].

\section{Summary}

Despite the profound incidence of PE and associated morbidity and mortality, there remains a dearth of rigorous data in this clinical landscape, leading to limitations in risk quantification, stratification, and therapeutic strategies. In the past decade, there have been advances in catheter-directed provision of thrombolytic therapy, novel therapies for thrombus degradation and percutaneous thrombus removal, and ongoing growth in multidisciplinary care utilization through the PERT model. The three currently FDA-cleared devices for acute $\mathrm{PE}$ received clearance predicated on single-arm prospective studies that utilized short-term clinical endpoints and surrogate markers for right ventricular dysfunction.

Aggregation and data collaboration to better understand patient- and therapy-related risks will require a concerted effort from PERT team members locally and the PERT Consortium nationally to provide expertise on minimizing harm while maximizing benefit of these novel therapies. Machine learning and artificial intelligence may play a larger role for risk stratification models in the future.

Availability of Data and Material All material included in this manuscript is publicly available information.

\section{Compliance with Ethical Standards}

Conflict of Interest Dr. Jay Giri serves on advisory boards for Astra Zeneca and Inari Medical, and Dr. Sameer Khandhar sit on the advisory board for Inari Medical. Dr. Connor Tice, Dr. Matthew Seigerman, Dr. Steven Pugliese, Dr. Paul Fiorilli, and Dr. Tai Kobayashi have no conflicts of interests to report.

Human and Animal Rights Informed Consent This article does not contain any studies with human or animal subjects performed by any of the authors.

\section{References}

Papers of particular interest, published recently, have been highlighted as:

- Of importance

- Of major importance

1. Blood clots: a serious but preventable medical condition. Centers for Disease Control and Prevention, (CDC) 2016 May, 4.

2. Office of the Surgeon General, (US), National Heart, Lung, and Blood Institute (US). The surgeon general's call to action to prevent deep vein thrombosis and pulmonary embolism. Office of the Surgeon General (US) 2008.

3. Sanchez O, Trinquart L, Colombet I, Durieux P, Huisman MV, Chatellier G, et al. Prognostic value of right ventricular dysfunction in patients with haemodynamically stable pulmonary embolism: a systematic review. Eur Heart J. 2008;29(12):1569-77.

4. Secemsky E, Chang Y, Jain CC, Beckman JA, Giri J, Jaff MR, et al. Contemporary management and outcomes of patients with massive and submassive pulmonary embolism. Am J Med. 2018;131(12): 1506-1514. e0.

5. O'Connell C. How I treat incidental pulmonary embolism. Blood. 2015;125(12):1877-82.

6. Pollack CV, Schreiber D, Goldhaber SZ, Slattery D, Fanikos J, O'Neil BJ, et al. Clinical characteristics, management, and outcomes of patients diagnosed with acute pulmonary embolism in the emergency department: initial report of EMPEROR (multicenter emergency medicine pulmonary embolism in the real world registry). J Am Coll Cardiol. 2011;57(6):700-6.

7. Jaff MR, McMurtry MS, Archer SL, Cushman M, Goldenberg N, Goldhaber SZ, et al. Management of massive and submassive pulmonary embolism, iliofemoral deep vein thrombosis, and chronic thromboembolic pulmonary hypertension: a scientific statement from the American Heart Association. Circulation. 2011;123(16): $1788-830$.

8. Konstantinides SV, Meyer G, Becattini C, Bueno H, Geersing G, Harjola V, et al. 2019 ESC guidelines for the diagnosis and management of acute pulmonary embolism developed in collaboration with the European Respiratory Society (ERS). Eur Respir J. 2019;54(3):1901647 Konstantinides et al. updates the previous ESC guidelines for the diagnosis and management of acute PE last published in 2014. The ESC guidelines outline on of the two major clinical classification systems for acute pulmonary embolism.

9. Vanni S, Nazerian P, Pepe G, Baioni M, Risso M, Grifoni G, et al. Comparison of two prognostic models for acute pulmonary embolism: clinical vs. right ventricular dysfunction-guided approach. J Thromb Haemost. 2011;9(10):1916-23.

10. Becattini C, Agnelli G. Predictors of mortality from pulmonary embolism and their influence on clinical management. Thromb Haemost. 2008;100(05):747-51.

11. Becattini C, Agnelli G, Lankeit M, Masotti L, Pruszczyk P, Casazza F, et al. Acute pulmonary embolism: mortality prediction by the 2014 European Society of Cardiology risk stratification model. Eur Respir J. 2016;48(3):780-6.

12. Chatterjee S, Chakraborty A, Weinberg I, Kadakia M, Wilensky RL, Sardar P, et al. Thrombolysis for pulmonary embolism and risk of all-cause mortality, major bleeding, and intracranial hemorrhage: a meta-analysis. JAMA. 2014;311(23):2414-21.

13. Jiménez D, Kopecna D, Tapson V, Briese B, Schreiber D, Lobo JL, et al. Derivation and validation of multimarker prognostication for normotensive patients with acute symptomatic pulmonary embolism. Am J Respir Crit Care Med. 2014;189(6):718-26. 
14. Subramaniam RM, Mandrekar J, Chang C, Blair D, Gilbert K, Peller PJ, et al. Pulmonary embolism outcome: a prospective evaluation of CT pulmonary angiographic clot burden score and ECG score. Am J Roentgenol. 2008;190(6):1599-604.

15. Furlan A, Aghayev A, Chang CH, Patil A, Jeon KN, Park B, et al. Short-term mortality in acute pulmonary embolism: clot burden and signs of right heart dysfunction at CT pulmonary angiography. Radiology. 2012;265(1):283-93.

16. Smith SB, Geske JB, Maguire JM, Zane NA, Carter RE, Morgenthaler TI. Early anticoagulation is associated with reduced mortality for acute pulmonary embolism. Chest. 2010;137(6): 1382-90.

17. Tu T, Toma C, Tapson VF, Adams C, Jaber WA, Silver M, et al. Appendix of a prospective, single-arm, multicenter trial of catheterdirected mechanical thrombectomy for intermediate-risk acute pulmonary embolism: the FLARE study. J Am Coll Cardiol Intv. 2019;12(9):859-69.

18. Marti C, John G, Konstantinides S, Combescure C, Sanchez O, Lankeit M, et al. Systemic thrombolytic therapy for acute pulmonary embolism: a systematic review and meta-analysis. Eur Heart J. 2014;36(10):605-14.

19. Meyer G, Vicaut E, Danays T, Agnelli G, Becattini C, BeyerWestendorf J, et al. Fibrinolysis for patients with intermediate-risk pulmonary embolism. N Engl J Med. 2014;370(15):1402-11.

20. Kearon C, Akl EA, Ornelas J, Blaivas A, Jimenez D, Bounameaux $\mathrm{H}$, et al. Antithrombotic therapy for VTE disease: CHEST guideline and expert panel report. Chest. 2016;149(2):315-52.

21. Kuo WT, Banerjee A, Kim PS, DeMarco FJ, Levy JR, Facchini FR, et al. Pulmonary embolism response to fragmentation, Embolectomy, and catheter thrombolysis (PERFECT): initial results from a prospective multicenter registry. Chest. 2015;148(3): 667-73.

22. Braaten JV, Goss RA, Francis CW. Ultrasound reversibly disaggregates fibrin fibers. Thromb Haemost. 1997;78(01):1063-8.

23. Kucher N, Boekstegers P, Müller OJ, Kupatt C, Beyer-Westendorf $\mathrm{J}$, Heitzer T, et al. Randomized, controlled trial of ultrasoundassisted catheter-directed thrombolysis for acute intermediate-risk pulmonary embolism. Circulation. 2014;129(4):479-86.

24. Piazza G, Hohlfelder B, Jaff MR, Ouriel K, Engelhardt TC, Sterling $\mathrm{KM}$, et al. A prospective, single-arm, multicenter trial of ultrasound-facilitated, catheter-directed, low-dose fibrinolysis for acute massive and submassive pulmonary embolism: the SEATTLE II study. J Am Coll Cardiol Intv. 2015;8(10):1382-92.

25. Liang NL, Avgerinos ED, Marone LK, Singh MJ, Makaroun MS, Chaer RA. Comparative outcomes of ultrasound-assisted thrombolysis and standard catheter-directed thrombolysis in the treatment of acute pulmonary embolism. Vasc Endovasc Surg. 2016;50(6):40510

26. Rao G, Xu H, Wang JJ, Galmer A, Giri J, Jaff MR, et al. Ultrasound-assisted versus conventional catheter-directed thrombolysis for acute pulmonary embolism: A multicenter comparison of patient-centered outcomes. Vasc Med. 2019;24(3):241-7 Rao et al. is a retrospective analysis over a three-year period for both clinical and quality-of-life outcomes for patients undergoing CDL or USAT. Over the study period no statistical difference was found between these groups based on clinical endpoints (RVSP) and safety endpoints of moderate to severe bleeding. In an analysis of patient-centered QOL outcomes, the authors found no significant difference between these two groups.

27. Graif A, Grilli CJ, Kimbiris G, Agriantonis DJ, Chohan OZ, Fedele $\mathrm{CR}$, et al. Comparison of ultrasound-accelerated versus pigtail catheter directed thrombolysis for the treatment of acute massive and submassive pulmonary embolism. J Vasc Interv Radiol. 2017;28(10):1339-47.
28. Tapson VF, Sterling K, Jones N, Elder M, Tripathy U, Brower J, et al. A randomized trial of the optimum duration of acoustic pulse thrombolysis procedure in acute intermediate-risk pulmonary embolism: the OPTALYSE PE Trial. J Am Coll Cardiol Intv. 2018;11(14):1401-10 Tapson et al. is a randomized prospective multicenter, parallel-group trial that randomized patients to four treatment groups in order to determine the lowest optimal tPA dose and treatment duration for USAT for patients with intermediate-risk PE. Analyses found that lower doses of therapy from 4 to $12 \mathrm{mg}$ per lung with infusion duration of 2 to $6 \mathrm{~h}$ resulted in improved right ventricular surrogate outcome endpoints (RV to $L V$ ratio) and clot burden compared with baseline metrics.

29. Chatterjee S, Weinberg I, Yeh RW, Chakraborty A, Sardar P, Weinberg MD, et al. Risk factors for intracranial haemorrhage in patients with pulmonary embolism treated with thrombolytic therapy Development of the PE-CH Score. Thromb Haemost. 2017;117(02):246-51.

30. Chatterjee S, Lip GY, Giri J. HAS-BLED versus ATRIA risk scores for intracranial hemorrhage in patients receiving thrombolytics for pulmonary embolism. J Am Coll Cardiol. 2016;67(24):2904-5.

31.• Giri J, Sista AK, Weinberg I, Kearon C, Kumbhani DJ, Desai ND, et al. Interventional therapies for acute pulmonary embolism: current status and principles for the development of novel evidence: a scientific statement From the American Heart Association. Circulation. 2019;140(20):e774-801 Giri et al. produced this 2019 scientific statement from the American Heart Association in order to both review the current endovascular interventional therapies for acute $\mathbf{P E}$ and to propose guidance for how new evidence should guide new device deployment into clinical practice. The authors review current risk stratification tools, risks, and benefits of current interventional therapies, and discuss the pros and cons of the PERT model.

32. Schissler AJ, Gylnn RJ, Sobieszczyk PS, Waxman AB. Ultrasound-assisted catheter-directed thrombolysis compared with anticoagulation alone for treatment of intermediate-risk pulmonary embolism. Pulm Circ. 2018;8(4):2045894018800265.

33. Ierardi AM, Xhepa G, Piffaretti G, Bacuzzi A, Tozzi M, Carbone $\mathrm{M}$, et al. Clinical experience with Angiojet: a comprehensive review. Int Angiol. 2015;34(6):1-14.

34. Chechi T, Vecchio S, Spaziani G, Giuliani G, Giannotti F, Arcangeli $\mathrm{C}$, et al. Rheolytic thrombectomy in patients with massive and submassive acute pulmonary embolism. Catheter Cardiovasc Interv. 2009;73(4):506-13.

35. Bonvini RF, Roffi M, Bounameaux H, Noble S, Müller H, Keller P, et al. AngioJet rheolytic thrombectomy in patients presenting with high-risk pulmonary embolism and cardiogenic shock: a feasibility pilot study. EuroIntervention. 2013;8(12):1419-27.

36. Lin PH, Okada T, Steinberg JL, Zhou W, El Sayed HF, Rawat A, et al. Rheolytic pharmacomechanical thrombectomy in experimental chronic deep vein thrombosis: effect of L-arginine on thrombogenicity and endothelial vasomotor function. World $\mathrm{J}$ Surg. 2007;31(4):664-75.

37. Tu T, Toma C, Tapson VF, Adams C, Jaber WA, Silver M, et al. A prospective, single-arm, multicenter trial of catheter-directed mechanical thrombectomy for intermediate-risk acute pulmonary embolism: the FLARE Study. J Am Coll Cardiol Intv. 2019;12(9): 859-69 $\mathrm{Tu}$ et al. was a single-arm prospective trial aimed at establishing the effectiveness and safety of the FlowTriever System. In this analysis of $\mathbf{1 0 6}$ patients, the authors showed that this system deployed in patients with acute intermediate-risk $P E$ led to a significant reduction in $R V / L V$ ratios with overall low observed bleeding rates. After publication of this trial the FlowTriever system received FDA $\mathbf{5 1 0}(\mathrm{k})$ clearance for treatment of PE. 
38. Donaldson CW, Baker JN, Narayan RL, Provias TS, Rassi AN, Giri JS, et al. Thrombectomy using suction filtration and veno-venous bypass: single center experience with a novel device. Catheter Cardiovasc Interv. 2015;86(2):E81-7.

39. Moriarty JM, Al-Hakim R, Bansal A, Park JK. Removal of caval and right atrial thrombi and masses using the AngioVac device: initial operative experience. J Vasc Interv Radiol. 2016;27(10): 1584-91.

40. Devcic Z, Kuo WT. Percutaneous pulmonary embolism thrombectomy and thrombolysis: technical tips and tricks. Semin Interv Radiol. 2018;35(02):129-35.

41. Ciampi-Dopazo JJ, Romeu-Prieto JM, Sánchez-Casado M, Romerosa B, Canabal A, Rodríguez-Blanco ML, et al. Aspiration thrombectomy for treatment of acute massive and submassive pulmonary embolism: initial single-center prospective experience. $\mathrm{J}$ Vasc Interv Radiol. 2018;29(1):101-6.

42. Al-Hakim R, Bhatt A, Benenati JF. Continuous aspiration mechanical thrombectomy for the management of submassive pulmonary embolism: a single-center experience. J Vasc Interv Radiol. 2017;28(10):1348-52.

43. Sista A. EXTRACT-PE an "important first study" for aspiration thrombectomy in acute PE patients. 2019.

44. Zuckerman B. 510(K) summary-FlowTriever retrieval/aspiration system Food and Drug Administration. 2018.

45. Corsi F, Lebreton G, Bréchot N, Hekimian G, Nieszkowska A, Trouillet $\mathrm{J}$, et al. Life-threatening massive pulmonary embolism rescued by venoarterial-extracorporeal membrane oxygenation. Crit Care. 2017;21(1):76.

46. Meneveau N, Guillon B, Planquette B, Piton G, Kimmoun A, Gaide-Chevronnay L, et al. Outcomes after extracorporeal membrane oxygenation for the treatment of high-risk pulmonary embolism: a multicentre series of 52 cases. Eur Heart J. 2018;39(47): 4196-204 Meneveau et al. performed a multicenter retrospective review of 52 cases of high-risk PE. With a primary outcome of all-cause 30-day mortality and a secondary outcome of 90day major bleeding, this study demonstrated a significant improvement in 30-day mortality with ECMO treatment. However, a significant portion of ECMO patients experienced an in-hospital major bleeding event.

47. Ain DL, Albaghdadi M, Giri J, Abtahian F, Jaff MR, Rosenfield K, et al. Extra-corporeal membrane oxygenation and outcomes in massive pulmonary embolism: two eras at an urban tertiary care hospital. Vasc Med. 2018;23(1):60-4.

48. Al-Bawardy R, Rosenfield K, Borges J, Young MN, Albaghdadi M, Rosovsky R, et al. Extracorporeal membrane oxygenation in acute massive pulmonary embolism: a case series and review of the literature. Perfusion. 2019;34(1):22-8.

49. Barnal AG, Fanola C, Bartos JA. Management of PE. 2020; Available at: https://www.acc.org/latest-in-cardiology/articles/ 2020/01/27/07/42/management-of-pe. Accessed 04/08/, 2020.

50. Kasper W, Konstantinides S, Geibel A, Olschewski M, Heinrich F, Grosser KD, et al. Management strategies and determinants of outcome in acute major pulmonary embolism: results of a multicenter registry. J Am Coll Cardiol. 1997;30(5):1165-71.

51. Dudzinski DM, Giri J, Rosenfield K. Interventional treatment of pulmonary embolism. Circ Cardiovasc Interv. 2017;10(2): e004345.

52. Barnes GD, Kabrhel C, Courtney DM, Naydenov S, Wood T, Rosovsky R, et al. Diversity in the pulmonary embolism response team model: an organizational survey of the national PERT consortium members. Chest. 2016;150(6):1414-7.

53. Barnes G, Giri J, Courtney DM, Naydenov S, Wood T, Rosovsky $\mathrm{R}$, et al. Nuts and bolts of running a pulmonary embolism response team: results from an organizational survey of the National PERT Consortium members. Hosp Pract. 2017;45(3):76-80.
54.• Rivera-Lebron B, McDaniel M, Ahrar K, Alrifai A, Dudzinski DM, Fanola C, et al. Diagnosis, treatment and follow up of acute pulmonary embolism: consensus practice from the PERT consortium. Clin Appl Thromb Hemost. 2019;25:1076029619853037 RiveraLebron et al. outline a comprehensive review document from the PERT consortium on management for acute PE. The authors combine both clinical data and consensus opinion to outline recommendations for the treatment of different clinical scenarios of PE management.

55. Todoran TM, Giri J, Barnes GD, Rosovsky RP, Chang Y, Jaff MR, et al. Treatment of submassive and massive pulmonary embolism: a clinical practice survey from the second annual meeting of the Pulmonary Embolism Response Team Consortium. J Thromb Thrombolysis. 2018;46(1):39-49.

56. Schoepf UJ, Kucher N, Kipfmueller F, Quiroz R, Costello P, Goldhaber SZ. Right ventricular enlargement on chest computed tomography: a predictor of early death in acute pulmonary embolism. Circulation. 2004;110(20):3276-80.

57. Sista AK, Goldhaber SZ, Vedantham S, Kline JA, Kuo WT, Kahn SR, et al. Research priorities in submassive pulmonary embolism: proceedings from a multidisciplinary research consensus panel. J Vasc Interv Radiol. 2016;27(6):787-94.

58. Banerjee I, Sofela M, Yang J, Chen JH, Shah NH, Ball R, et al. Development and performance of the Pulmonary Embolism Result Forecast Model (PERFORM) for computed tomography clinical decision support. JAMA Netw Open. 2019;2(8):e198719 Banerjee et al. aimed to develop a machine learning model capable of creating a patient-specific risk score for $\mathrm{PE}$. The authors concluded that the PERFORM can consider patientspecific risk factors and dependent variables to create a PE risk prediction. This prediction can then generalize to new population sets. The authors suggest that this may be a tool applicable for patients referred for CT PE as a way to improve CT use.

59. Jain CC, Chang Y, Kabrhel C, Giri J, Channick R, RodriguezLopez J, et al. Impact of pulmonary arterial clot location on pulmonary embolism treatment and outcomes (90 days). Am J Cardiol. 2017;119(5):802-7.

60. Liu W, Liu M, Guo X, Zhang P, Zhang L, Zhang R, et al. Evaluation of acute pulmonary embolism and clot burden on CTPA with deep learning. Eur Radiol. 2020;1-9.

61. Kumar BP, Rajasekhar D, Vanajakshamma V. Study of clinical, radiological and echocardiographic features and correlation of Qanadli CT index with RV dysfunction and outcomes in pulmonary embolism. Indian Heart J. 2014;66(6):629-34.

62. Apfaltrer P, Henzler T, Meyer M, Roeger S, Haghi D, Gruettner J, et al. Correlation of $\mathrm{CT}$ angiographic pulmonary artery obstruction scores with right ventricular dysfunction and clinical outcome in patients with acute pulmonary embolism. Eur J Radiol. 2012;81(10):2867-71.

63. Zahoor A, Khan N, Khan G. Correlation of Qanadli score with right ventricular dysfunction in patients with acute pulmonary embolism. Pak J Phys. 2017;13(4):14-7.

64. Klok FA, Kruip M, Van der Meer N, Arbous MS, Gommers D, Kant KM, et al. Incidence of thrombotic complications in critically ill ICU patients with COVID-19. Thromb Res. 2020. https://doi. org/10.1016/j.thromres.2020.04.013.

65. Bikdeli B, Madhavan MV, Jimenez D, Chuich T, Dreyfus I, Driggin E, et al. COVID-19 and thrombotic or thromboembolic disease: implications for prevention, antithrombotic therapy, and follow-up. J Am Coll Cardiol. 2020;75(23):2950-2973.

Publisher's Note Springer Nature remains neutral with regard to jurisdictional claims in published maps and institutional affiliations. 